\title{
A NEW GENERALIZATION OF POWER LINDLEY DISTRIBUTION: WITH APPLICATIONS TO LIFETIME DATA
}

\author{
MAHMOUD M. MANSOUR ${ }^{1}$ and MOHAMED S. HAMED ${ }^{2}$ \\ 1,2Department of Statistics \\ Mathematics and Insurance \\ Benha University \\ Egypt \\ e-mail: Mahmoud.mansour@fcom.bu.edu.eg \\ 2Department of Business Administration \& Technologies \\ Taibah University \\ Saudi Arabia \\ e-mail:moswilem@gmail.com
}

\begin{abstract}
This paper introduces a new generalization of the power Lindley distribution, based on a new family of lifetime distribution by Mansour et al. [19]. We refer to the new distribution as new generalization of the power Lindley (NGPL) distribution. The new model contains some of lifetime distributions as special cases such as power Lindley, transmuted Lindley, and Lindley distributions. The properties of the new model are discussed and the maximum likelihood estimation is used to evaluate the parameters. Explicit expressions are derived for the moments and examine the order statistics. It will be shown that the analytical results are applicable to model real data.
\end{abstract}

2010 Mathematics Subject Classification: 60-XX, 60EXX.

Keywords and phrases: transmutation, survival function, exponentiated exponential, order statistics, maximum likelihood estimation.

Received March 3, 2015

(ㄷ) 2015 Scientific Advances Publishers 


\section{Introduction}

The quality of the procedures used in a statistical analysis depends heavily on the assumed probability model or distributions. Because of it, considerable effort has been expended in the development of large classes of standard probability distributions along with relevant statistical methodologies. However, there still remain many important problems where the real data does not follow any of the classical or standard probability models. Since there is a clear need for extended forms of these distributions, a significant progress has been made toward the generalization of some well-known distributions and their successful application to problems in areas such as engineering, finance, economics and biomedical sciences, among others.

For complex electronic and mechanical systems, the failure rate often exhibits non-monotonic (bathtub or upside-down bathtub unimodal) failure rates (Xie and Lai [30]). Distributions with such failure rates have attracted a considerable attention of researchers in reliability engineering. In software reliability, bathtub shaped failure rate is encountered in firmware, and in embedded software in hardware devices. Firmware plays an important role in functioning of hard drives of large computers, spacecraft and high performance aircraft control systems, advanced weapon systems, safety critical control systems used for monitoring the industrial process in chemical and nuclear plants (Zhang et al. [31]). The upside down bathtub shaped failure rate is used in data of motor bus failures (Mudholkar et al. [24]), for optimal burn-in decisions (Block and Savits [6]), Chang [7], for ageing properties in reliability (Gupta and Gupta [14], Jiang et al. [16] and the course of a disease whose mortality reaches a peak after some finite period and then declines gradually.

Lindley [18], introduced a one parameter distribution, known as Lindley distribution with parameter $\theta$ if its probability density is defined as 


$$
f(x)=\frac{\theta^{2}}{\theta+1}(1+x) e^{-\theta x}, \quad x>0, \theta>0 .
$$

The corresponding cumulative distribution function (cdf) is:

$$
F(x)=1-\frac{\theta+1+\theta x}{\theta+1} e^{-\theta x}, \quad x>0, \theta>0 .
$$

Ghitany et al. [11] have discussed various properties of this distribution and showed that in many ways that the pdf given by (1) provides a better model for some applications than the exponential distribution. Bakouch et al. [4] obtained an extended Lindley distribution and discussed its various properties and applications. Ghitany et al. [12] developed a two parameters weighted Lindley distribution and discussed its applications to survival data. Nadarajah et al. [25] obtained a generalized Lindley distribution and discussed its various properties and applications. Merovci and Elbatal [22] use the quadratic rank transmutation map in order to generate a flexible family of probability distributions taking Lindley geometric distribution as the base value distribution by introducing a new parameter that would offer more distributional flexibility and called it transmuted Lindley geometric distribution. Asgharzadeh et al. [3] introduced a general family of continuous lifetime distributions by compounding any continuous distribution and the Poisson Lindley distribution. Oluyede and Yang [26] proposed a new four-parameter class of generalized Lindley distribution (GLD) called the beta-generalized Lindley distribution (BGLD). This class of distributions contains the beta Lindley, GLD, and Lindley distributions as special cases. A two parameter power Lindley distribution (PLD), of which the Lindley distribution 'Equation (1)' is a particular case, has been suggested by Ghitany et al. [10]. They introduced a new extension of the Lindley distribution called power Lindley distribution by its probability density function pdf

$$
f(x)=\frac{\theta^{2} \beta x^{\beta-1}}{\theta+1}\left(1+x^{\beta}\right) e^{-\theta x^{\beta}}, \quad x>0, \theta>0 .
$$


The corresponding cumulative distribution function (cdf) is:

$$
F(x)=1-\frac{\theta+1+\theta x^{\beta}}{\theta+1} e^{-\theta x^{\beta}}, \quad x>0, \theta>0 .
$$

An interesting idea of generalizing a distribution, known in the literature by transmutation, is derived by using the quadratic rank transmutation map (QRTM) introduced by Shaw and Buckley [28]. Merovci [20] introduced transmuted Lindley distribution. According to the transmutation generalization approach, the cdf satisfies the relationship

$$
F(x)=(1+\lambda) G(x)-\lambda[G(x)]^{2},
$$

where $G(x)$ the cdf of the baseline distribution.

In this article, we use new transmutation map approach suggested by Mansour et al. [19] to define a new model which power Lindley distribution. The proposed modification generalizes the rank of the transmutation map by replacing the constant power by additional parameters. The following definition gives the mechanism of generating a new family of lifetime distributions building on a base model, that is, according to this modification.

Definition 1.1. Let $G(x)$ be the cumulative distribution function (cdf) of a nonnegative absolutely continuous random variable, $G(x)$ be strictly increasing on its support, and $G(0)=0$ define a new cdf, $F(x)$, out of $G(x)$ as

$$
F(x)=(1+\lambda)[G(x)]^{\delta}-\lambda[G(x)]^{\alpha}, \quad x>0,
$$

where $\alpha, \delta>0$ if $0>\lambda>-1$ and $\alpha>0,(\alpha+\alpha / 4) \geq \delta \geq(\alpha / 2)$ if $0<\lambda<1$.

The rest of the article is organized as follows. In Section 2, introduces the proposed new generalization of the power Lindley model according to the new class of distribution. In Section 3, we find the reliability function, 
hazard rate and cumulative hazard rate of the subject model. The expansion for the pdf and the cdf functions is derived in Section 4. In Section 5, the statistical properties include quantile functions, median, moments, and moment generating function are given. In Section 6, order statistics are discussed. In Section 7, we introduce the method of likelihood estimation as point estimation and, give the equation used to estimate the parameters, using the maximum product spacing estimates and the least square estimates techniques. Finally, we fit the distribution to real data set to examine it.

\section{A New Transmuted Lindley Distribution}

The new generalization of the power Lindley (NGPL) distribution, now using (6) and (4), we have the cdf of a new distribution

$$
F(x)=(1+\lambda)\left[1-\frac{\theta+1+\theta x^{\beta}}{\theta+1} e^{-\theta x^{\beta}}\right]^{\delta}-\lambda\left[1-\frac{\theta+1+\theta x^{\beta}}{\theta+1} e^{-\theta x^{\beta}}\right]^{\alpha}, x>0
$$

Hence, the pdf of new generalization of the power Lindley distribution is

$$
\begin{gathered}
f(x)=\frac{\theta^{2} \beta x^{\beta-1}}{\theta+1}\left(1+x^{\beta}\right) e^{-\theta x^{\beta}}\left((1+\lambda) \delta\left[1-\frac{\theta+1+\theta x^{\beta}}{\theta+1} e^{-\theta x^{\beta}}\right]^{\delta-1}\right. \\
\left.-\lambda \alpha\left[1-\frac{\theta+1+\theta x^{\beta}}{\theta+1} e^{-\theta x^{\beta}}\right]^{\alpha-1}\right),
\end{gathered}
$$

where $\theta, \alpha, \delta>0$, if $0>\lambda>-1$, and $\theta, \alpha>0,(\alpha+\alpha / 4) \geq \delta \geq(\alpha / 2)$ if $0<\lambda<1$.

We present special cases of the new generalization of the power Lindley (NGPL) distribution as follows: 
Power Lindley distribution: For $\lambda=0$, and $\delta=1$, the distribution function (7) becomes

$$
F(x)=1-\frac{\theta+1+\theta x^{\beta}}{\theta+1} e^{-\theta x^{\beta}}, \quad x>0,
$$

which is the distribution function of the power Lindley distribution.

Transmuted Lindley distribution: For $\alpha=2, \beta=1$ and $\delta=1$, the distribution function (7) becomes

$$
F(x)=(1+\lambda)\left[1-\frac{\theta+1+\theta x}{\theta+1} e^{-\theta x}\right]-\lambda\left[1-\frac{\theta+1+\theta x}{\theta+1} e^{-\theta x}\right]^{2}, \quad x>0,
$$

which is the distribution function of the transmuted Lindley distribution.

Transmuted exponentiated Lindley distribution: For $\beta=1$ and $\delta=\frac{\alpha}{2}$, the distribution function (7) becomes

$$
F(x)=(1+\lambda)\left[1-\frac{\theta+1+\theta x}{\theta+1} e^{-\theta x}\right]^{\frac{\alpha}{2}}-\lambda\left[1-\frac{\theta+1+\theta x}{\theta+1} e^{-\theta x}\right]^{\alpha}, x>0
$$

which is the distribution function of the transmuted exponentiated Lindley distribution.

Exponentiated Lindley distribution: For $\lambda=0$, and $\beta=1$, the distribution function (7) becomes

$$
F(x)=\left[1-\frac{\theta+1+\theta x}{\theta+1} e^{-\theta x}\right]^{\delta}, \quad x>0,
$$

which is the distribution function of the exponentiated Lindley distribution.

Lindley distribution: For $\lambda=0, \beta=1$ and $\delta=1$, the distribution function (7) becomes 


$$
F(x)=\left[1-\frac{\theta+1+\theta x}{\theta+1} e^{-\theta x}\right], \quad x>0
$$

which is the distribution function of the Lindley distribution.

Transmuted power Lindley distribution: For $\alpha=2$, and $\delta=1$, the distribution function (7) becomes

$$
F(x)=(1+\lambda)\left[1-\frac{\theta+1+\theta x^{\beta}}{\theta+1} e^{-\theta x^{\beta}}\right]-\lambda\left[1-\frac{\theta+1+\theta x^{\beta}}{\theta+1} e^{-\theta x^{\beta}}\right]^{2}, \quad x>0
$$

which is the distribution function of the transmuted Lindley distribution.

Exponentiated power Lindley distribution: For $\lambda=0$, the distribution function (7) becomes

$$
F(x)=\left[1-\frac{\theta+1+\theta x^{\beta}}{\theta+1} e^{-\theta x^{\beta}}\right]^{\delta}, \quad x>0,
$$

which is the distribution function of the exponentiated power Lindley distribution.

Transmuted exponentiated power Lindley distribution: For $\delta=\frac{\alpha}{2}$, the distribution function (7) becomes

$F(x)=(1+\lambda)\left[1-\frac{\theta+1+\theta x^{\beta}}{\theta+1} e^{-\theta x^{\beta}}\right]^{\frac{\alpha}{2}}-\lambda\left[1-\frac{\theta+1+\theta x^{\beta}}{\theta+1} e^{-\theta x^{\beta}}\right]^{\alpha}, x>0$

which is the distribution function of the transmuted exponentiated Lindley distribution. 
Figures 1 and 2 illustrates some of the possible shapes of the pdf and cdf of the generalization of the power Lindley distribution the parameters $\lambda, \theta, \delta$

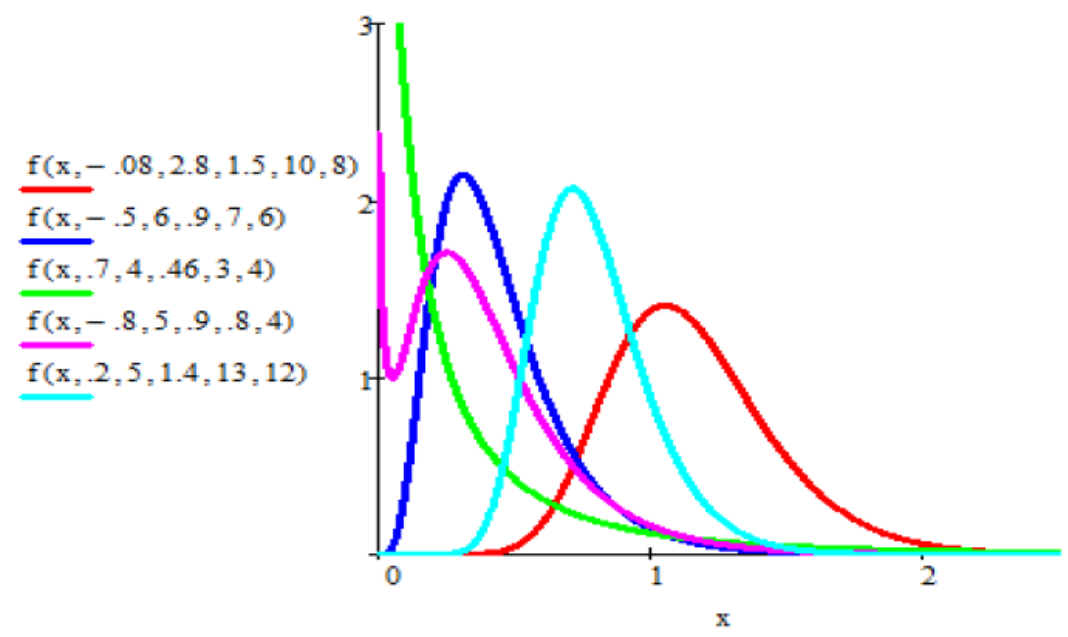

Figure 1. Probability density function of the NGPL distribution.

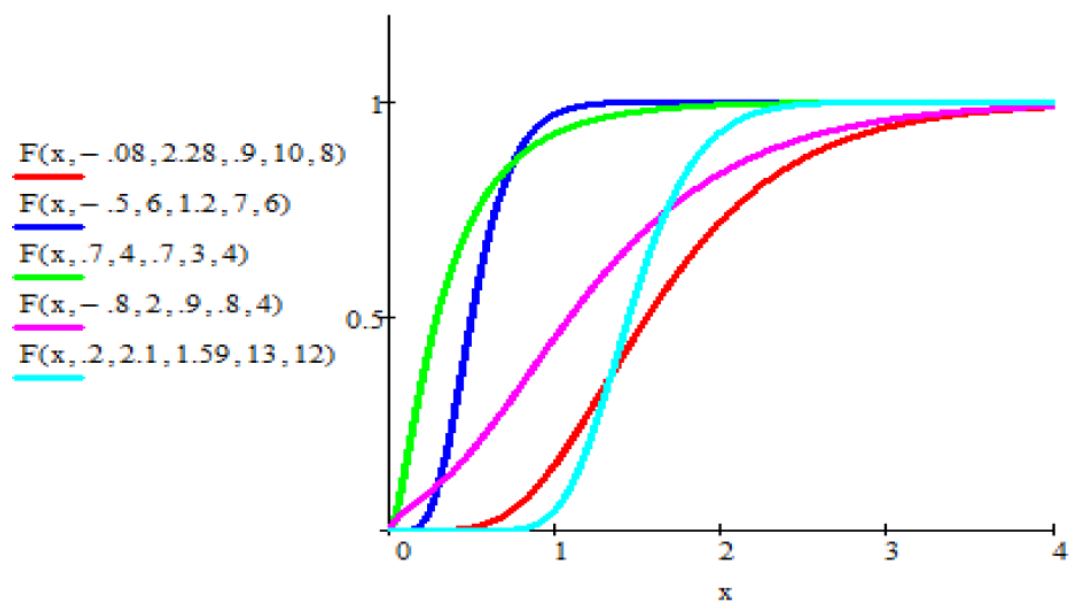

Figure 2. Distributionf of the NGPL distribution. 


\section{Reliability Analysis}

The characteristics in reliability analysis which are the reliability function $(\mathrm{RF})$, the hazard rate function ( $\mathrm{HF})$, and the cumulative hazard rate function $(\mathrm{CHF})$ for the NGPL are introduces in this section.

\subsection{Reliability function}

The reliability function (RF) also known as the survival function, which is the probability of an item not failing prior to some time $t$, is defined by $R(x)=1-F(x)$. The reliability function of the new generalization of the power lindley distribution (NGPL) denoted by

$R_{N G P N}(\lambda, \theta, \beta, \delta, \alpha)$, can be a useful characterization of life time data analysis. It can be defined as

$$
R_{N G P L}(\lambda, \theta, \beta, \delta, \alpha)=1-F_{N G P L}(\lambda, \theta, \beta, \delta, \alpha),
$$

the survival function of is given by

$$
\begin{aligned}
& R_{N G P L}(x, \lambda, \theta, \beta, \delta, \alpha) \\
& \quad=1-\left[(1+\lambda)\left[1-\frac{\theta+1+\theta x^{\beta}}{\theta+1} e^{-\theta x^{\beta}}\right]^{\delta}-\lambda\left[1-\frac{\theta+1+\theta x^{\beta}}{\theta+1} e^{-\theta x^{\beta}}\right]^{\alpha}\right] .
\end{aligned}
$$

Figure 3 illustrates the pattern of the called the new generalization of the power Lindley distribution reliability function with different choices of parameters $\lambda, \theta, \beta, \delta$, and $\alpha$. 


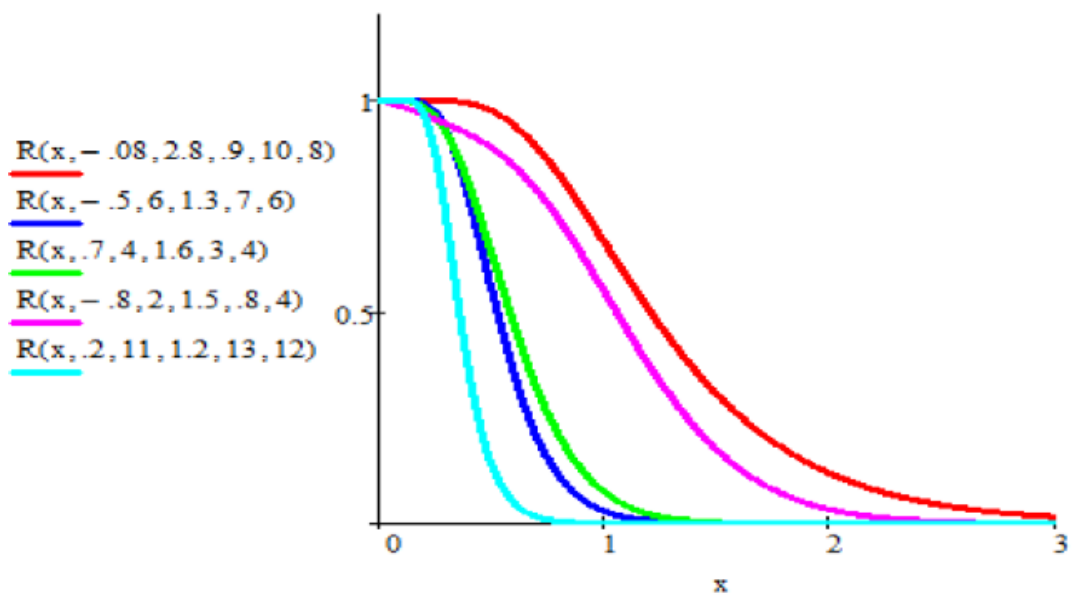

Figure 3. Reliability function of the NGPL distribution.

\subsection{Hazard rate function}

The other characteristic of interest of a random variable is the hazard rate function $(\mathrm{HF})$. The new generalization of the power Lindley distribution also known as instantaneous failure rate denoted by $h_{N G P L}(x)$, is an important quantity characterizing life phenomenon. It can be loosely interpreted as the conditional probability of failure, given it has survived to the time $t$. The HF of the NGPL is defined by $h_{N G P L}(x, \lambda, \theta, \beta, \delta, \alpha)=f_{N G N G}(x, \lambda, \theta, \beta, \delta, \alpha) / R_{N G P L}(x, \lambda, \theta, \beta, \delta, \alpha)$.

Figure 4 illustrates some of the possible shapes of the hazard rate function of the new generalization of the power Lindley distribution for different values of the parameters $\lambda, \theta, \beta, \delta$, and $\alpha$. 


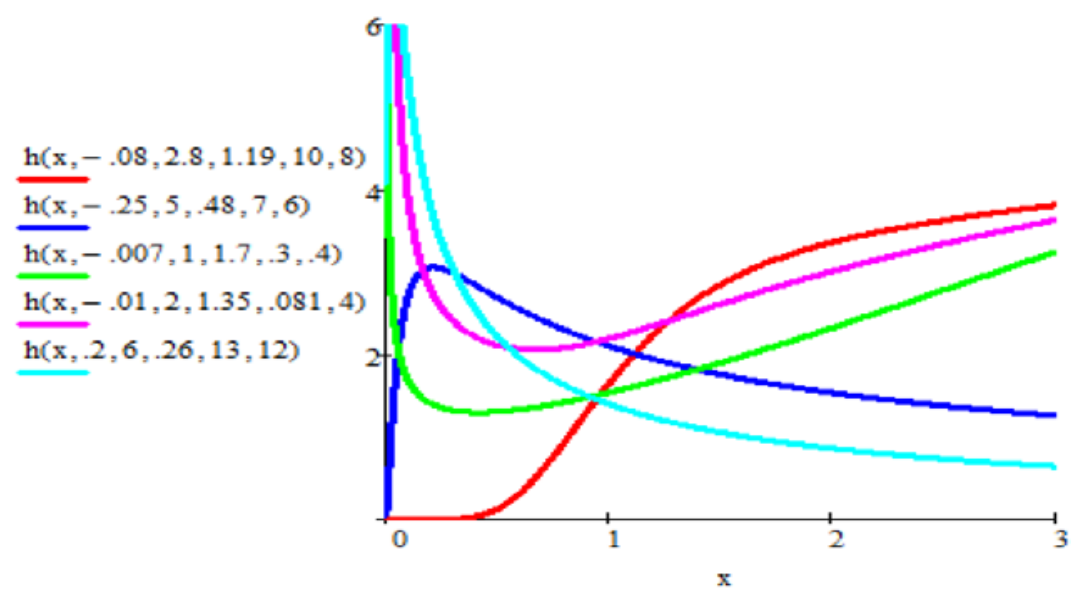

Figure 4. Hazard rate of the NGPL distribution.

The NGPL model due to its flexibility in accommodating all forms of the hazard rate function as seen from Figure 4 (by changing its parameter values) seems to be an important distribution that can be used.

\subsection{Cumulative hazard rate function}

The cumulative hazard function (CHF) of the new generalization of the power Lindley distribution, denoted by $H_{N G P L}(x, \lambda, \theta, \beta, \delta, \alpha)$, is defined as

$$
\begin{aligned}
H_{N G P L}(x, \lambda, \theta, \beta, \delta, \alpha) & =\int_{0}^{x} h_{N G P L}(x, \lambda, \theta, \beta, \delta, \alpha) d x \\
& =-\ln R_{N G P L}(x, \lambda, \theta, \beta, \delta, \alpha), \\
H_{N T L}(x, \lambda, \theta, \delta, \alpha)=- & \ln \left(1-\left[(1+\lambda)\left[1-\frac{\theta+1+\theta x^{\beta}}{\theta+1} e^{-\theta x^{\beta}}\right]^{\delta}\right.\right. \\
& \left.\left.-\lambda\left[1-\frac{\theta+1+\theta x^{\beta}}{\theta+1} e^{-\theta x^{\beta}}\right]^{\alpha}\right]\right) .
\end{aligned}
$$




\section{Expansion for the pdf and the cdf Functions}

In this section, we introduced another expression for the pdf and the cdf functions using. The Maclaurin expansion to simplifying the pdf and the cdf forms.

\subsection{Expansion for the pdf function}

From Equation (8) and using the expansions

$$
(1-z)^{k}=\sum_{j=0}^{\infty} \frac{(-1)^{j} \Gamma(k+1)}{\Gamma(k-j+1) j !} z^{j}
$$

which holds for $|z|<1$ and $k>0$, and

$$
(a-b)^{k}=\sum_{j=0}^{k}\left(\begin{array}{l}
k \\
j
\end{array}\right)(-1)^{j} b^{j} a^{k-j}
$$

Using (19) and applying it to (8), the pdf of the NGPL model can be written as

$$
\begin{gathered}
f(x)=\frac{\theta^{2} \beta x^{\beta-1}}{\theta+1}\left(1+x^{\beta}\right) e^{-\theta x^{\beta}} \sum_{i=0}^{1}(-1)^{i} \lambda^{i} \alpha^{i}\left[1-\frac{\theta+1+\theta x^{\beta}}{\theta+1} e^{-\theta x^{\beta}}\right]^{i(\alpha-1)+(\delta-1)(1-i)} \\
\times(1+\lambda)^{1-i} \delta^{1-i}
\end{gathered}
$$

Using (19) and applying it to (21), the pdf of the NGPL model can be written as

$$
\begin{gathered}
f(x)=\frac{\theta^{2} \beta x^{\beta-1}}{\theta+1}\left(1+x^{\beta}\right) e^{-\theta x^{\beta}} \sum_{i=0}^{1} \sum_{j=0}^{\infty} \frac{(-1)^{i+j} \Gamma(i(\alpha-1)+(\delta-1)(1-i)+1)}{j ! \Gamma(i(\alpha-1)+(\delta-1)(1-i)-j+1)} \\
\times\left(\lambda^{i} \alpha^{i}(1+\lambda)^{1-i} \delta^{1-i}\left(\frac{\theta+1+\theta x^{\beta}}{\theta+1}\right)^{j} e^{-\theta j x^{\beta}}\right)
\end{gathered}
$$

the pdf of the NGPL model can be written as 


$$
\begin{gathered}
f(x)=\frac{\theta^{2} \beta x^{\beta-1}}{\theta+1} \sum_{i=0}^{1} \sum_{j=0}^{\infty} \sum_{k=0}^{j} \frac{(-1)^{i+j} \Gamma(i(\alpha-1)+(\delta-1)(1-i)+1) \theta^{k}}{k ! \Gamma(j-k+1) \Gamma(i(\alpha-1)+(\delta-1)(1-i)-j+1)(\theta+1)^{j}} \\
\times\left(\lambda^{i} \alpha^{i}(1+\lambda)^{1-i} \delta^{1-i}\left(1+x^{\beta}\right)^{k+1} e^{-\theta x^{\beta}}(j+1)\right), \\
f(x)=\frac{\theta^{2} \beta}{\theta+1} \sum_{i=0}^{1} \sum_{j=0}^{\infty} \sum_{k=0}^{j} \sum_{l=0}^{k+1} \frac{(-1)^{i+j} \Gamma(i(\alpha-1)+(\delta-1)(1-i)+1) k \theta^{k}}{l ! \Gamma(k-l+2) \Gamma(j-k+1) \Gamma(i(\alpha-1)+(\delta-1)(1-i)-j+1)(\theta+1)^{j}} \\
\times\left(\lambda^{i} \alpha^{i}(1+\lambda)^{1-i} \delta^{1-i} x^{\beta(l+1)-1} e^{-\theta x^{\beta}(j+1)}\right),
\end{gathered}
$$

the pdf of NGPL distribution can then be represented as

$$
f(x)=\sum_{i=0}^{1} \sum_{j=0}^{\infty} \sum_{k=0}^{j} \sum_{l=0}^{k+1} A_{i: l} \times\left(x^{\beta(l+1)-1} e^{-\beta x^{\beta}(j+1)}\right),
$$

where $A_{i: l}$ is a constant term given by

$$
A_{i: l}=\frac{\theta^{2} \beta}{\theta+1} \times \frac{(-1)^{i+j} \Gamma(i(\alpha-1)+(\delta-1)(1-i)+1) k \theta^{k} \lambda^{i} \alpha^{i}(1+\lambda)^{1-i} \delta^{1-i}}{l ! \Gamma(k-l+2) \Gamma(j-k+1) \Gamma(i(\alpha-1)+(\delta-1)(1-i)-j+1)(\theta+1)^{j}} .
$$

\subsection{Expansion for the cdf function}

Using expansion (19) and (20) to Equation (7), then the cdf function of the NGPL can be written as

$$
F(x)=\sum_{i=0}^{1} \sum_{j, m=0}^{\infty} \sum_{k=0}^{j} \sum_{l=0}^{k} B_{i: l} x^{\beta(l+m)}
$$

where $B_{i: l}$ is a constant term given by

$$
B_{i: l}=\frac{(-1)^{i+j+m} \lambda^{i}(1-\lambda)^{1-i} \Gamma(\alpha i+\delta(1-i)+1) \theta^{k+m} j^{m}}{l ! m ! \Gamma(\alpha i+\delta(1-i)-j+1) \Gamma(j-k+1) \Gamma(k-l+1)(1+\theta)^{j}} .
$$

\section{Statistical Properties}

In this section, we discuss the most important statistical properties of the NGPL distribution. 


\subsection{Quantile function}

The quantile function is obtained by inverting the cumulative distribution (26), where the $p$-th quantile $x_{p}$ of the NGPL model is the real solution of the following equation:

$$
\sum_{i=0}^{1} \sum_{j, m=0}^{\infty} \sum_{k=0}^{j} \sum_{l=0}^{k} B_{i: l} x_{p}^{\beta(l+m)}-p=0 .
$$

An expansion for the median $M$ follows by taking $p=0.5$.

\subsection{Moments}

The $r$-th non-central moments or (moments about the origin) of the NGPL under using Equation (25) is given by

$$
\begin{gathered}
\mu_{r}^{\prime}=E\left(X^{r}\right)=\int_{0}^{\infty} X^{r} f(x) d x, \\
\mu_{r}^{\prime}=\int_{0}^{\infty} X^{r}\left[\sum_{i=0}^{1} \sum_{j=0}^{\infty} \sum_{k=0}^{j} \sum_{l=0}^{k+1} A_{i: l} \times\left(x^{\beta(l+1)-1} e^{-\theta x^{\beta}(j+1)}\right)\right] d x,
\end{gathered}
$$

then

$$
\mu_{r}^{\prime}=\sum_{i=0}^{1} \sum_{j=0}^{\infty} \sum_{k=0}^{j} \sum_{l=0}^{k+1} \frac{A_{i: l} \Gamma\left(\frac{r+\beta(l+1)}{\beta}\right)}{(\theta(j+1))^{\frac{r+\beta(l+1)}{\beta}}} .
$$

In particular, when $r=1$, Equation (27) yields the mean of the NGPL distribution, $\mu$ as

$$
\mu=\sum_{i=0}^{1} \sum_{j=0}^{\infty} \sum_{k=0}^{j} \sum_{l=0}^{k+1} \frac{A_{i: l} \Gamma\left(\frac{\beta(l+1)+1}{\beta}\right.}{(\theta(j+1))^{\frac{\beta(l+1)+1}{\beta}}} .
$$

The $n$-th central moments or (moments about the mean) can be obtained easily from the $r$-th non-central moments throw the relation 


$$
m_{u}=E(X-\mu)^{n}=\sum_{r=0}^{n}(-\mu)^{n-r} E\left(X^{r}\right) .
$$

Then the $n$-th central moments of the NGPL is given by

$$
m_{u}=E(X-\mu)^{n}=\sum_{r=0}^{n}(-\mu)^{n-r} \sum_{i=0}^{1} \sum_{j=0}^{\infty} \sum_{k=0}^{j} \sum_{l=0}^{k+1} \frac{A_{i: l} \Gamma\left(\frac{r+\beta(l+1)}{\beta}\right)}{(\theta(j+1))^{\frac{r+\beta(l+1)}{\beta}}} .
$$

\subsection{The moment generating function}

The moment generating function, $M_{x}(t)$, can be easily obtained from the $r$-th non-central moment through the relation

$$
M_{x}(t)=\sum_{r=0}^{\infty} \frac{t^{r}}{r !} \mu_{r}^{\prime}
$$

or

$$
\begin{gathered}
M_{x}(t)=\int_{0}^{\infty} e^{t x} f(x) d x \\
M_{x}(t)=\int_{0}^{\infty} e^{t x}\left[\sum_{i=0}^{1} \sum_{j=0}^{\infty} \sum_{k=0}^{j} \sum_{l=0}^{k+1} A_{i: l} \times\left(x^{\beta(l+1)-1} e^{-\theta x^{\beta}(j+1)}\right)\right] d x .
\end{gathered}
$$

Then, the moment generating function of the NGPL distribution is given by

$$
M_{x}(t)=\int_{0}^{\infty}\left[\sum_{i=0}^{1} \sum_{j, m=0}^{\infty} \sum_{k=0}^{j} \sum_{l=0}^{k+1} A_{i: l} \frac{t^{m}}{m !}\left(x^{m+\beta(l+1)-1} e^{-\theta x^{\beta}(j+1)}\right)\right] d x .
$$

Then, the moment generating function of the NGPL distribution is given by

$$
M_{x}(t)=\sum_{i=0}^{1} \sum_{j, m=0}^{\infty} \sum_{k=0}^{j} \sum_{l=0}^{k+1} A_{i: l} \frac{t^{m}}{m !}\left(\frac{\Gamma\left(\frac{m+\beta(l+1)}{\beta}\right)}{(\theta(j+1))^{\frac{m+\beta(l+1)}{\beta}}}\right)
$$




\section{Order Statistics}

Let $X_{1}, X_{2}, \ldots, X_{n}$ denote $n$-independent random variables from a distribution function $F_{X}(x)$ with pdf $f_{X}(x)$. Let $X_{(1)}, X_{(2)}, \ldots, X_{(n)}$ be the ordered sample arrangement. The pdf of $X_{(j)}$ is given by

$$
f_{X_{(j)}}(x)=\frac{n !}{(j-1) !(n-j) !} f_{X}(x)\left[F_{X}(x)\right]^{j-1} \cdot\left[1-F_{X}(x)\right]^{n-j}, j=1,2, \ldots, n .
$$

Then from (7) and (8), the pdf of $X_{(j)}$ is given by

$$
\begin{aligned}
f(x)=\frac{n !}{(j-1) !(n-j) !} \frac{\theta^{2} \beta x^{\beta-1}}{\theta+1}\left(1+x^{\beta}\right) e^{-\theta x^{\beta}}\left(( 1 + \lambda ) \delta \left[I(x, \theta]^{\delta-1}\right.\right. \\
-\lambda \alpha\left[I(x, \theta]^{\alpha-1}\right) \times\left[(1+\lambda)[I(x, \theta)]^{\delta}-\lambda[I(x, \theta)]^{\alpha}\right]^{j-1} \\
\times\left[1-\left((1+\lambda)[I(x, \theta)]^{\delta}-\lambda[I(x, \theta)]^{\alpha}\right)\right]^{n-j}
\end{aligned}
$$

where $I(x, \theta)=1-\frac{\theta+1+\theta x^{\beta}}{\theta+1} e^{-\theta x^{\beta}}$.

Therefore, the pdfs of the smallest and the largest order statistic are, respectively, given by

$$
\begin{aligned}
f_{X_{(1)}}(x)= & n \frac{\theta^{2} \beta x^{\beta-1}}{\theta+1}\left(1+x^{\beta}\right) e^{-\theta x^{\beta}}\left((1+\lambda) \delta[I(x, \theta)]^{\delta-1}-\lambda \alpha[I(x, \theta)]^{\alpha-1}\right) \\
& \times\left[1-\left((1+\lambda)[I(x, \theta)]^{\delta}-\lambda[I(x, \theta)]^{\alpha}\right)\right]^{n-1},
\end{aligned}
$$

and

$$
\begin{aligned}
f_{X_{(n)}}(x)= & n \frac{\theta^{2} \beta x^{\beta-1}}{\theta+1}\left(1+x^{\beta}\right) e^{-\theta x^{\beta}}\left((1+\lambda) \delta[I(x, \theta)]^{\delta-1}-\lambda \alpha[I(x, \theta)]^{\alpha-1}\right) \\
& \times\left[(1+\lambda)[I(x, \theta)]^{\delta}-\lambda[I(x, \theta)]^{\alpha}\right]^{n-1} .
\end{aligned}
$$




\section{Estimation of the Parameters}

In this section, we introduce the method of likelihood to estimate the parameters involved, then gives the equation used to estimate the parameters using the maximum product spacing estimates and the least square estimates techniques.

\subsection{Maximum likelihood estimation}

The maximum likelihood estimators (MLEs) for the parameters of the new generalization of the power Lindley distribution NGPL $(\lambda, \theta, \beta, \alpha, \delta)$ is discussed in this section. Consider the random sample $x_{1}, x_{2}, \ldots, x_{n}$ of size $n$ from NGPL $(\lambda, \theta, \beta, \alpha, \delta)$ with probability density function in (8), then the likelihood function can be expressed as follows:

$$
\begin{gathered}
L\left(x_{1}, x_{2}, \ldots, x_{n}, \lambda, \theta, \beta, \alpha, \delta\right)=\prod_{i=1}^{n} f_{N G P L}\left(x_{i}, \lambda, \theta, \beta, \alpha, \delta\right), \\
L\left(x_{1}, x_{2}, \ldots, x_{n}, \lambda, \theta, \beta, \alpha, \delta\right) \\
=\prod_{i=1}^{n} \frac{\theta^{2} \beta x_{i}^{\beta-1}}{\theta+1}\left(1+x_{i}^{\beta}\right) e^{-\theta x^{\beta}}\left((1+\lambda) \delta\left[I\left(x_{i}, \theta\right)\right]^{\delta-1}-\lambda \alpha\left[I\left(x_{i}, \theta\right)\right]^{\alpha-1}\right) .
\end{gathered}
$$

Hence, the log-likelihood function $\tau=\ln L$ becomes

$$
\begin{aligned}
\tau=2 n \ln \theta & -n \ln (\theta+1)+n \ln \beta+(\beta-1) \sum_{i=1}^{n} x_{i}+\sum_{i=1}^{n} \ln \left(1+x_{i}{ }^{\beta}\right)-\sum_{i=1}^{n} \theta x_{i}{ }^{\beta} \\
& +\sum_{i=1}^{n} \ln \left[(1+\lambda) \delta\left[I\left(x_{i}, \theta\right)\right]^{\delta-1}-\lambda \alpha\left[I\left(x_{i}, \theta\right)\right]^{\alpha-1}\right]
\end{aligned}
$$

where $I\left(x_{i}, \theta\right)=1-\frac{\theta+1+\theta x_{i}^{\beta}}{\theta+1} e^{-\theta x_{i}{ }^{\beta}}$.

Differentiating Equation (28) with respect to $\lambda, \theta, \beta, \delta$, and $\alpha$, then equating it to zero, we obtain the MLEs of $\lambda, \theta, \beta, \delta$, and $\alpha$ as follows: 


$$
\begin{aligned}
\frac{\partial \tau}{\partial \lambda}= & \sum_{i=1}^{n} \frac{\delta\left[I\left(x_{i}, \theta\right)\right]^{\delta-1}-\alpha\left[I\left(x_{i}, \theta\right)\right]^{\alpha-1}}{(1+\lambda) \delta\left[I\left(x_{i}, \theta\right)\right]^{\delta-1}-\lambda \alpha\left[I\left(x_{i}, \theta\right)\right]^{\alpha-1}}, \\
\frac{\partial \tau}{\partial \theta}= & \frac{2 n}{\theta}-\frac{n}{\theta+1}-\sum_{i=1}^{n} x_{i}{ }^{\beta} \\
& +\sum_{i=1}^{n} x_{i}^{\beta} e^{-\theta x_{i}{ }^{\beta}}\left[\left(1+\frac{\theta x_{i}^{\beta}}{\theta+1}\right)-\left(\frac{1}{(\theta+1)^{2}}\right)\right] \\
& \times \frac{\left(\delta(\delta-1)(1+\lambda)\left[I\left(x_{i}, \theta\right)\right]^{\delta-2}-\alpha(\alpha-1) \lambda\left[I\left(x_{i}, \theta\right)\right]^{\alpha-2}\right)}{(1+\lambda) \delta\left[I\left(x_{i}, \theta\right)\right]^{\delta-1}-\lambda \alpha\left[I\left(x_{i}, \theta\right)\right]^{\alpha-1}},
\end{aligned}
$$

$$
\begin{aligned}
\frac{\partial \tau}{\partial \beta}= & \frac{n}{\beta}+\sum_{i=1}^{n} x_{i}+\sum_{i=1}^{n} \frac{x_{i}{ }^{\beta} \ln \left(x_{i}\right)}{1+x_{i}{ }^{\beta}} \\
& +\sum_{i=1}^{n} \frac{\theta e^{-\theta x_{i}{ }^{\beta} x_{i}{ }^{\beta} \ln \left(x_{l}\right)}}{\theta+1}\left[\left(\theta+\theta x_{i}{ }^{\beta}+2\right)\right] \\
& \times \frac{\left(\delta(\delta-1)(1+\lambda)\left[I\left(x_{i}, \theta\right)\right]^{\delta-2}-\alpha(\alpha-1) \lambda\left[I\left(x_{i}, \theta\right)\right]^{\alpha-2}\right)}{(1+\lambda) \delta\left[I\left(x_{i}, \theta\right)\right]^{\delta-1}-\lambda \alpha\left[I\left(x_{i}, \theta\right)\right]^{\alpha-1}},
\end{aligned}
$$

$$
\frac{\partial \tau}{\partial \delta}=\sum_{i=1}^{n} \frac{(1+\lambda)\left[I\left(x_{i}, \theta\right)\right]^{\delta-1}\left(\delta \ln \left(I\left(x_{i}, \theta\right)\right)+1\right)}{(1+\lambda) \delta\left[I\left(x_{i}, \theta\right)\right]^{\delta-1}-\lambda \alpha\left[I\left(x_{i}, \theta\right)\right]^{\alpha-1}},
$$

and

$$
\frac{\partial \tau}{\partial \alpha}=\sum_{i=1}^{n} \frac{-\lambda\left[I\left(x_{i}, \theta\right)\right]^{\alpha-1}\left(\alpha \ln \left(I\left(x_{i}, \theta\right)\right)+1\right)}{(1+\lambda) \delta\left[I\left(x_{i}, \theta\right)\right]^{\delta-1}-\lambda \alpha\left[I\left(x_{i}, \theta\right)\right]^{\alpha-1}} .
$$

We can find the estimates of the unknown parameters by maximum likelihood method by setting these above nonlinear system of Equations (29)-(33) to zero and solve them simultaneously. These solutions will 
yield the ML estimators $\hat{\lambda}, \hat{\theta}, \hat{\beta}, \hat{\delta}$, and $\hat{\alpha}$. For the five parameters new generalization of the power Lindley distribution NGPL $(x, \lambda, \theta, \beta, \alpha, \delta) p d f$ all the second order derivatives exist. Thus we have the inverse dispersion matrix is given by

$$
\begin{gathered}
\left.\left(\begin{array}{c}
\hat{\lambda} \\
\hat{\theta} \\
\hat{\beta} \\
\hat{\delta} \\
\hat{\alpha}
\end{array}\right) \sim N\left[\begin{array}{l}
\lambda \\
\theta \\
\beta \\
\delta \\
\alpha
\end{array}\right),\left(\begin{array}{lllll}
\widehat{V}_{11} & \widehat{V}_{12} & \widehat{V}_{13} & \widehat{V}_{14} & \widehat{V}_{15} \\
\widehat{V}_{21} & \widehat{V}_{22} & \widehat{V}_{23} & \widehat{V}_{24} & \widehat{V}_{25} \\
\widehat{V}_{31} & \widehat{V}_{32} & \widehat{V}_{33} & \widehat{V}_{34} & \widehat{V}_{35} \\
\widehat{V}_{41} & \widehat{V}_{42} & \widehat{V}_{43} & \widehat{V}_{44} & \widehat{V}_{45} \\
\widehat{V}_{51} & \widehat{V}_{52} & \widehat{V}_{53} & \widehat{V}_{54} & \widehat{V}_{55}
\end{array}\right)\right], \\
V^{-1}=-E\left(\begin{array}{lllll}
V_{11} & V_{12} & V_{13} & V_{14} & V_{15} \\
V_{21} & V_{22} & V_{23} & V_{24} & V_{25} \\
V_{31} & V_{32} & V_{33} & V_{34} & V_{35} \\
V_{41} & V_{42} & V_{43} & V_{44} & V_{45} \\
V_{51} & V_{52} & V_{53} & V_{54} & V_{55}
\end{array}\right) .
\end{gathered}
$$

Equation (34) is the variance covariance matrix of the NGPL $(x, \lambda, \theta, \beta, \alpha, \delta)$, where

$$
\begin{aligned}
& V_{11}=\frac{\partial^{2} \psi}{\partial \lambda^{2}}, \quad V_{12}=\frac{\partial^{2} \psi}{\partial \lambda \partial \theta}, \quad V_{13}=\frac{\partial^{2} \psi}{\partial \lambda \partial \beta}, \quad V_{14}=\frac{\partial^{2} \psi}{\partial \lambda \partial \delta}, \quad V_{15}=\frac{\partial^{2} \psi}{\partial \lambda \partial \alpha^{\prime}} \\
& V_{22}=\frac{\partial^{2} \psi}{\partial \theta^{2}}, \quad V_{23}=\frac{\partial^{2} \psi}{\partial \theta \partial \beta}, \quad V_{24}=\frac{\partial^{2} \psi}{\partial \theta \partial \delta}, \quad V_{25}=\frac{\partial^{2} \psi}{\partial \theta \partial \alpha^{\prime}}, \\
& V_{33}=\frac{\partial^{2} \psi}{\partial \beta^{2}}, \quad V_{34}=\frac{\partial^{2} \psi}{\partial \beta \partial \delta}, \quad V_{35}=\frac{\partial^{2} \psi}{\partial \beta \partial \alpha^{\prime}}, \\
& V_{44}=\frac{\partial^{2} \psi}{\partial \delta^{2}}, \quad V_{45}=\frac{\partial^{2} \psi}{\partial \delta \partial \alpha}, \\
& V_{55}=\frac{\partial^{2} \psi}{\partial \alpha^{2}} .
\end{aligned}
$$


By solving this inverse dispersion matrix, these solutions will yield the asymptotic variance and covariances of these MLEs for $\hat{\lambda}, \hat{\theta}, \hat{\beta}, \hat{\delta}$, and $\hat{\alpha}$. Approximate $100(1-\phi) \%$ confidence intervals for $\lambda, \theta, \delta$, and $\alpha$ can be determined as

$$
\hat{\lambda} \pm Z_{\frac{\phi}{2}} \sqrt{\hat{V}_{11}}, \hat{\theta} \pm Z_{\frac{\phi}{2}} \sqrt{\hat{V}_{22}}, \hat{\beta} \pm Z_{\frac{\phi}{2}} \sqrt{\hat{V}_{33}}, \hat{\delta} \pm Z_{\frac{\phi}{2}} \sqrt{\hat{V}_{44}} \text {, and } \hat{\alpha} \pm Z_{\frac{\phi}{2}} \sqrt{\hat{V}_{55}} \text {, }
$$

where $Z_{\frac{\phi}{2}}$ is the upper $\phi$-th percentile of the standard normal distribution.

\subsection{Maximum product spacing estimates}

The maximum product spacing (MPS) method has been proposed by Cheng and Amin [8]. This method is based on an idea that the differences (spacing) of the consecutive points should be identically distributed. The geometric mean of the differences is given as

$$
G M=\sqrt[n+1]{\prod_{i=1}^{n+1} D_{i}}
$$

where, the difference $D_{i}$ is defined as

$$
D_{i}=\int_{x_{(i-1)}}^{x_{(i)}} f(x, \lambda, \theta, \beta, \delta, \alpha) d x ; \quad i=1,2, \ldots, n+1
$$

where $F\left(x_{(0)}, \lambda, \theta, \beta, \delta, \alpha\right)=0$ and $F\left(x_{(n+1)}, \lambda, \theta, \beta, \delta, \alpha\right)=0$. The MPS estimators $\hat{\lambda}_{P S}, \hat{\theta}_{P S}, \hat{\beta}_{P S}, \hat{\alpha}_{P S}$, and $\hat{\delta}_{P S}$ of $\lambda, \theta, \beta, \alpha$, and $\delta$ are obtained by maximizing the geometric mean (GM) of the differences. Substituting pdf of NGPL distribution in (36) and taking logarithm of the above expression, we will have

$$
\log G M=\frac{1}{n+1} \sum_{i=1}^{n+1} \log \left[F\left(x_{(i)}, \lambda, \theta, \beta, \delta, \alpha\right)-F\left(x_{(i-1)}, \lambda, \theta, \beta, \delta, \alpha\right)\right] .
$$


The MPS estimators $\hat{\lambda}_{P S}, \hat{\theta}_{P S}, \hat{\beta}_{P S}, \hat{\delta}_{P S}$, and $\hat{\alpha}_{P S}$ of $\lambda, \theta, \beta, \delta$, and $\alpha$ can be obtained as the simultaneous solution of the following nonlinear equations:

$$
\begin{aligned}
& \frac{\partial \log G M}{\partial \lambda}=\frac{1}{n+1} \sum_{i=1}^{n+1}\left[\frac{F_{\lambda}^{\prime}\left(x_{(i)}, \lambda, \theta, \beta, \delta, \alpha\right)-F_{\lambda}^{\prime}\left(x_{(i-1)}, \lambda, \theta, \beta, \delta, \alpha\right)}{F\left(x_{(i)}, \lambda, \theta, \beta, \delta, \alpha\right)-F\left(x_{(i-1)}, \lambda, \theta, \beta, \delta, \alpha\right)}\right]=0, \\
& \frac{\partial \log G M}{\partial \theta}=\frac{1}{n+1} \sum_{i=1}^{n+1}\left[\frac{F_{\theta}^{\prime}\left(x_{(i)}, \lambda, \theta, \beta, \delta, \alpha\right)-F_{\theta}^{\prime}\left(x_{(i-1)}, \lambda, \theta, \beta, \delta, \alpha\right)}{F\left(x_{(i)}, \lambda, \theta, \beta, \delta, \alpha\right)-F\left(x_{(i-1)}, \lambda, \theta, \beta, \delta, \alpha\right)}\right]=0, \\
& \frac{\partial \log G M}{\partial \beta}=\frac{1}{n+1} \sum_{i=1}^{n+1}\left[\frac{F_{\beta}^{\prime}\left(x_{(i)}, \lambda, \theta, \beta, \delta, \alpha\right)-F_{\beta}^{\prime}\left(x_{(i-1)}, \lambda, \theta, \beta, \delta, \alpha\right)}{F\left(x_{(i)}, \lambda, \theta, \beta, \delta, \alpha\right)-F\left(x_{(i-1)}, \lambda, \theta, \beta, \delta, \alpha\right)}\right]=0, \\
& \frac{\partial \log G M}{\partial \delta}=\frac{1}{n+1} \sum_{i=1}^{n+1}\left[\frac{F_{\delta}^{\prime}\left(x_{(i)}, \lambda, \theta, \beta, \delta, \alpha\right)-F_{\delta}^{\prime}\left(x_{(i-1)}, \lambda, \theta, \beta, \delta, \alpha\right)}{F\left(x_{(i)}, \lambda, \theta, \beta, \delta, \alpha\right)-F\left(x_{(i-1)}, \lambda, \theta, \beta, \delta, \alpha\right)}\right]=0,
\end{aligned}
$$

and

$$
\frac{\partial \log G M}{\partial \alpha}=\frac{1}{n+1} \sum_{i=1}^{n+1}\left[\frac{F_{\alpha}^{\prime}\left(x_{(i)}, \lambda, \theta, \delta, \alpha\right)-F_{\alpha}^{\prime}\left(x_{(i-1)}, \lambda, \theta, \delta, \alpha\right)}{F\left(x_{(i)}, \lambda, \theta, \delta, \alpha\right)-F\left(x_{(i-1)}, \lambda, \theta, \delta, \alpha\right)}\right]=0 .
$$

\subsection{Least square estimates}

Let $x_{(1)}, x_{(2)}, \ldots, x_{(n)}$ be the ordered sample of size $n$ drawn the NGPL distribution. Then, the expectation of the empirical cumulative distribution function is defined as

$$
E\left[F\left(X_{(i)}\right)\right]=\frac{i}{n+1} ; \quad i=1,2, \ldots, n .
$$

The least square estimates $\hat{\lambda}_{L S}, \hat{\theta}_{L S}, \hat{\beta}_{L S}, \hat{\delta}_{L S}$, and $\hat{\alpha}_{L S}$ of $\lambda, \theta, \beta, \delta$, and $\alpha$ are obtained by minimizing

$$
Z(\lambda, \theta, \beta, \delta, \alpha)=\sum_{i=1}^{n}\left[F\left(x_{(i)}, \lambda, \theta, \beta, \delta, \alpha\right)-\frac{i}{n+1}\right]^{2}
$$


Therefore, $\hat{\lambda}_{L S}, \hat{\theta}_{L S}, \hat{\beta}_{L S}, \hat{\delta}_{L S}$, and $\hat{\alpha}_{L S}$ of $\lambda, \theta, \beta, \delta$, and $\alpha$ can be obtained as the solution of the following system of equations:

$$
\begin{aligned}
& \frac{\partial Z(\lambda, \theta, \beta, \delta, \alpha)}{\partial \lambda}=\sum_{i=1}^{n} F_{\lambda}^{\prime}\left(x_{(i)}, \lambda, \theta, \beta, \delta, \alpha\right)\left(F\left(x_{(i)}, \lambda, \theta, \beta, \delta, \alpha\right)-\frac{i}{n+1}\right)=0 \\
& \frac{\partial Z(\lambda, \theta, \beta, \delta, \alpha)}{\partial \theta}=\sum_{i=1}^{n} F_{\theta}^{\prime}\left(x_{(i)}, \lambda, \theta, \beta, \delta, \alpha\right)\left(F\left(x_{(i)}, \lambda, \theta, \beta, \delta, \alpha\right)-\frac{i}{n+1}\right)=0 \\
& \frac{\partial Z(\lambda, \theta, \beta, \delta, \alpha)}{\partial \beta}=\sum_{i=1}^{n} F_{\beta}^{\prime}\left(x_{(i)}, \lambda, \theta, \beta, \delta, \alpha\right)\left(F\left(x_{(i)}, \lambda, \theta, \beta, \delta, \alpha\right)-\frac{i}{n+1}\right)=0 \\
& \frac{\partial Z(\lambda, \theta, \beta, \delta, \alpha)}{\partial \delta}=\sum_{i=1}^{n} F_{\delta}^{\prime}\left(x_{(i)}, \lambda, \theta, \beta, \delta, \alpha\right)\left(F\left(x_{(i)}, \lambda, \theta, \beta, \delta, \alpha\right)-\frac{i}{n+1}\right)=0
\end{aligned}
$$

and

$$
\frac{\partial Z(\lambda, \theta, \beta, \delta, \alpha)}{\partial \alpha}=\sum_{i=1}^{n} F_{\alpha}^{\prime}\left(x_{(i)}, \lambda, \theta, \beta, \delta, \alpha\right)\left(F\left(x_{(i)}, \lambda, \theta, \beta, \delta, \alpha\right)-\frac{i}{n+1}\right)=0 .
$$

These non-linear can be routinely solved by using Newton's method or fixed point iteration techniques. The subroutines to solve non-linear optimization problem are available in R, software namely optim( ), $\mathrm{nlm}($ ), and bbmle( ) etc. We used nlm( ) package for optimizing (28).

\section{Application}

In this section, we use a real data set to show that the new generalization of the power Lindley distribution can be a better model than one based on the Lindley distribution. The data set represents an uncensored data set corresponding to remission times (in months) of a random sample of 128 bladder cancer patients reported in Merovci [20]. Some summary statistics for the data are as follows: 


$\begin{array}{cccccc}\text { Min } & \text { 1st Qu } & \text { Median } & \text { Mean } & \text { 3rd Qu } & \text { Max. } \\ 0.080 & 3.348 & 6.395 & 9.366 & 11.840 & 79.05\end{array}$

In order to compare the two distribution models, we consider criteria like KS (Kolmogorov-Smirnov), -2L , AIC (Akaike information criterion), and AICC (corrected Akaike information criterion) for the data set. The better distribution corresponds to smaller $\mathrm{KS},-2 \mathcal{L}$, $\mathrm{AIC}$, and $\mathrm{AICC}$ values:

$$
\begin{gathered}
\mathrm{AIC}=-2 \mathcal{L}+2 k, \\
\mathrm{AIC}_{C}=-2 \mathcal{L}+\left(\frac{2 k n}{n-k-1}\right),
\end{gathered}
$$

where $\mathcal{L}$ denotes the log-likelihood function evaluated at the maximum likelihood estimates, $k$ is the number of parameters, and $n$ is the sample size.

Also, for calculating the values of KS, we use the sample estimates of $\lambda, \theta, \beta, \delta$, and $\alpha$. Table 1 shows the parameter estimation based on the maximum likelihood and least square estimation, and gives the values of the criteria AIC, AICC, and KS test. The values in Table 1 indicate that the NGPL distribution is a strong competitor to other distributions used here for fitting data. 
Table 1. MLEs the measures AIC, AICC, and KS test to data for the models.

\begin{tabular}{|c|c|c|c|c|c|c|}
\hline Model & $\begin{array}{l}\text { Parameter } \\
\text { Estimates }\end{array}$ & $\begin{array}{l}\text { Standard } \\
\text { Error }\end{array}$ & $-2 \mathrm{LL}$ & $\mathrm{AIC}$ & $\mathrm{AIC}_{\mathrm{C}}$ & $\mathrm{KS}$ \\
\hline New & $\lambda=-0.85835$ & 0.0938 & \multirow[t]{5}{*}{408.966} & \multirow[t]{5}{*}{827.932} & \multirow[t]{5}{*}{828.424} & \multirow[t]{5}{*}{0.02476} \\
\hline Transmuted & $\theta=2.5044$ & 1.6574 & & & & \\
\hline \multirow[t]{3}{*}{ Lindley } & $\beta=0.32916$ & 0.13408 & & & & \\
\hline & $\delta=6.67978$ & 2.6466 & & & & \\
\hline & $\alpha=33.7380$ & 15.584 & & & & \\
\hline Power & $\theta=0.29432$ & 0.03706 & \multirow[t]{2}{*}{413.353} & \multirow[t]{2}{*}{830.707} & \multirow[t]{2}{*}{830.803} & \multirow[t]{2}{*}{0.06822} \\
\hline Lindley & $\beta=0.83020$ & 0.04722 & & & & \\
\hline Transmuted & $\lambda=0.61687$ & 0.1688 & \multirow[t]{2}{*}{415.155} & \multirow[t]{2}{*}{834.3101} & \multirow[t]{2}{*}{834.4061} & \multirow[t]{2}{*}{0.226523} \\
\hline Lindley & $\theta=0.1557$ & 0.0150 & & & & \\
\hline Exponentiated & $\alpha=0.1648$ & 0.01664 & \multirow[t]{2}{*}{416.285} & \multirow[t]{2}{*}{936.5719} & \multirow[t]{2}{*}{836.6679} & \multirow[t]{2}{*}{0.092791} \\
\hline Lindley & $\theta=0.733$ & 0.0912 & & & & \\
\hline Lindley & $\theta=0.1960$ & 0.01234 & 419.529 & 841.0598 & 841.0916 & 0.116398 \\
\hline Weighted & $\alpha=0.15945$ & 0.0172 & \multirow[t]{2}{*}{416.442} & \multirow[t]{2}{*}{836.8845} & \multirow[t]{2}{*}{836.9805} & \multirow[t]{2}{*}{0.092567} \\
\hline Lindley & $\theta=0.6827$ & 0.1115 & & & & \\
\hline Modified & $\theta=6.2675$ & 3.16122 & \multirow[t]{3}{*}{413.969} & \multirow[t]{3}{*}{833.9393} & \multirow[t]{3}{*}{834.1329} & \multirow[t]{3}{*}{0.073875} \\
\hline \multirow[t]{2}{*}{ Weibull } & $\delta=6.3551$ & 3.1869 & & & & \\
\hline & $\alpha=1.001$ & 0.0017 & & & & \\
\hline
\end{tabular}




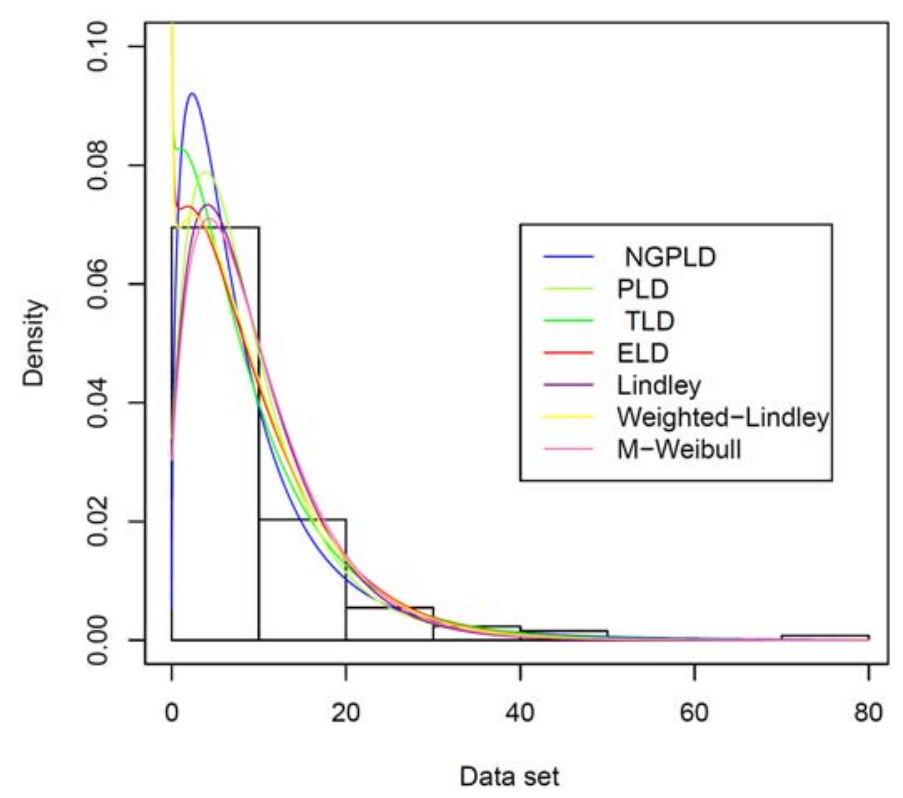

Figure 5. Estimated densities of data set.

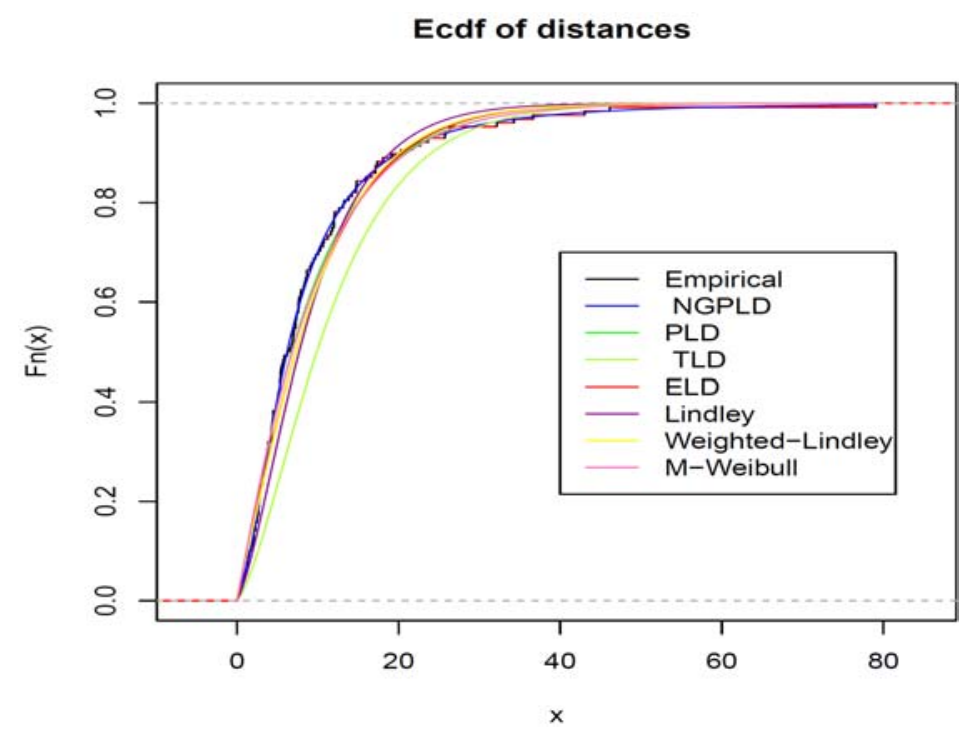

Figure 6. Empirical, fitted NGPL, power Lindley, transmuted Lindley, exponentiated Lindley, Lindley, weighted Lindley, and modified Weibull distributions of data set. 
58 MAHMOUD M. MANSOUR and MOHAMED S. HAMED
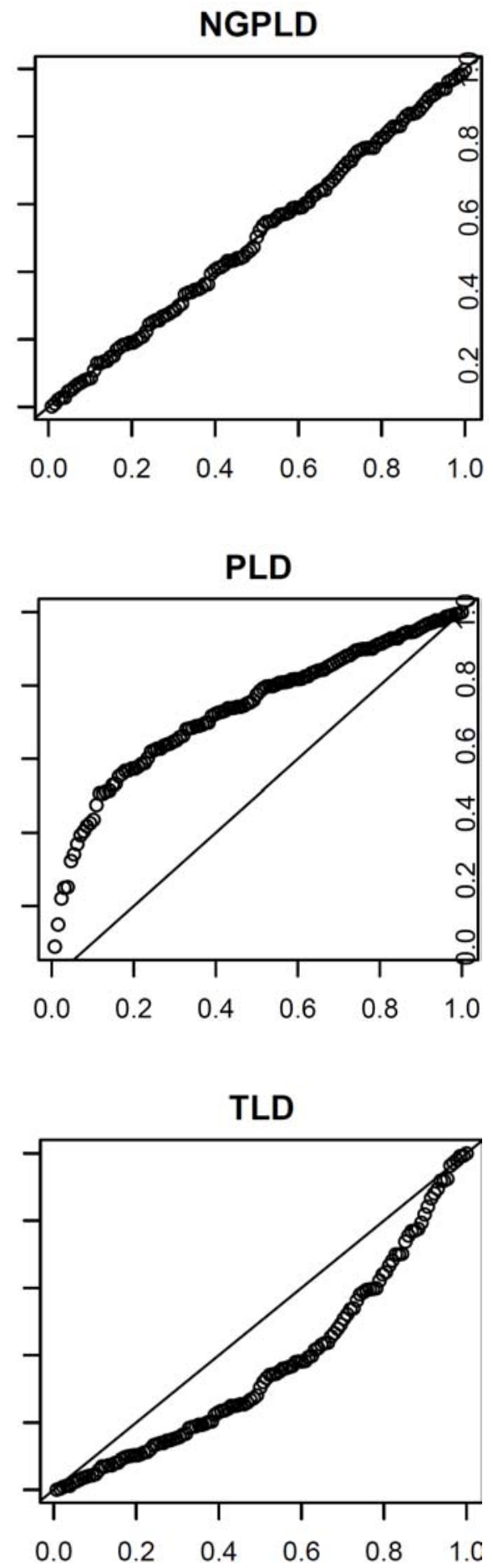


\section{ELD}

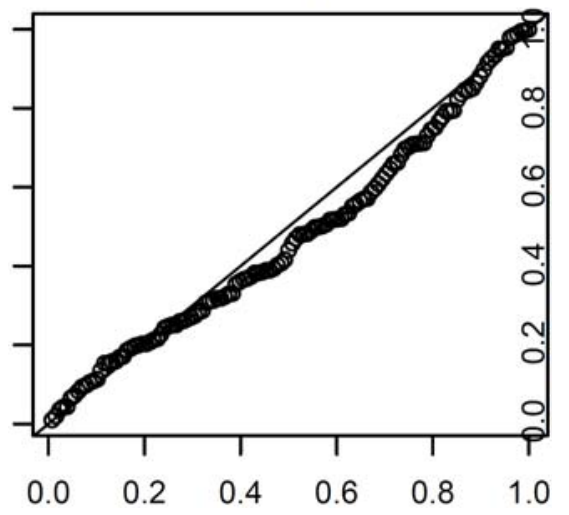

Lindley

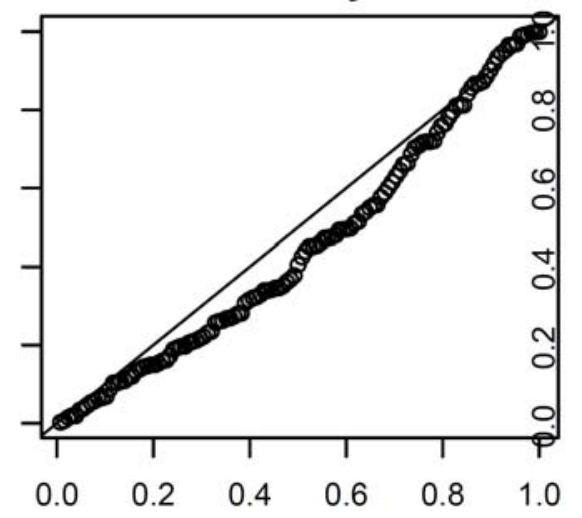

Weighted Lindley

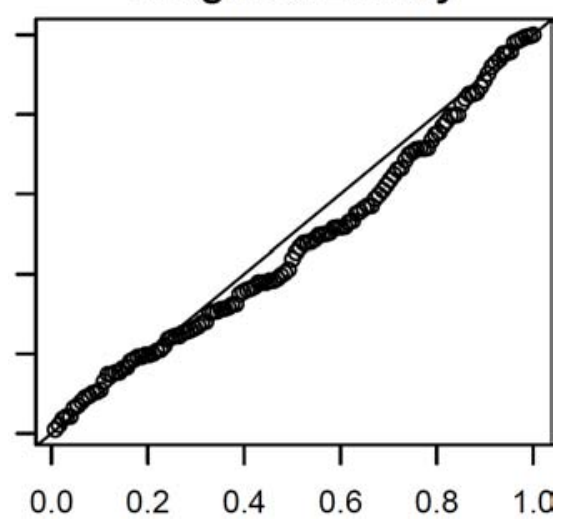




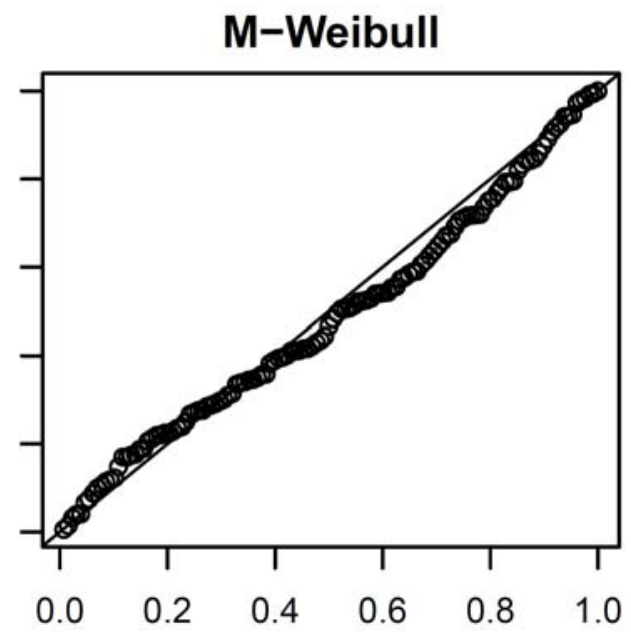

Figure 7. Probability plots for the fits NGPL, power Lindley, transmuted Lindley, exponentiated Lindley, Lindley, weighted Lindley, and modified Weibull distributions of data set.

\section{Simulation Algorithms}

In this section, we give an algorithm, using $\mathrm{R}$ software, to simulate data from the NGPL model.

\subsection{Inverse CDF method}

Since the probability integral transformation cannot be applied explicitly, we, therefore need to follow the following steps for generating a sample of size $n$ from NGPL $(\lambda, \theta, \beta, \delta, \alpha)$ :

Step 1. Set $n, \lambda, \theta, \beta, \delta, \alpha$ and initial value $x^{0}$.

Step 2. Generate $U \sim$ Uniform $(0,1)$.

Step 3. Update $x^{0}$ by using the Newton's formula.

$$
x^{*}=x^{0}-R\left(x^{0}, \theta\right),
$$


where $R\left(x^{0}, \theta\right)=\frac{F_{X}\left(x^{0}, \theta\right)-U}{F_{X}\left(x^{0}, \theta\right)}, F_{X}($.$) and f_{X}($.$) are cdf and pdf of$ NGPL distribution, respectively.

Step 4. If $\left|x^{0}-x^{*}\right| \leq \epsilon$, (very small, $\varepsilon>0$ tolerance limit), then store $x=x^{*}$ as a sample from NGPL distribution.

Step 5. If $\left|x^{0}-x^{*}\right|>\epsilon$, then set $x^{0}=x^{*}$ and go to Step 3 .

Step 6. Repeat Steps 3-5, $n$ times for $x_{1}, x_{2}, \ldots, x_{n}$, respectively.

\subsection{Inverse CDF method}

This subsection explores the behaviours of the proposed estimators in terms of their mean square error on the basis of simulated samples from pdf of NGPL with varying sample sizes. We take $\lambda=-0.55, \theta=1, \beta=3$, $\alpha=2$ arbitrarily and $n=10(10) 100$. The algorithms are coded in $\mathrm{R}$, and the algorithm given in 8.1 has been used for simulation purposes. We calculate MLE estimators of $\lambda, \theta, \beta, \delta$, and $\alpha$ based on each generated sample. This simulation is repeated 1000 of times, and average estimates with corresponding mean square errors are computed and reported in Table 2. 
Table 2. Estimates and mean square errors (in 2-nd row of each cell) of the proposed estimators with varying sample size.

\begin{tabular}{|c|c|c|c|c|c|}
\hline \multirow{2}{*}{$n$} & \multicolumn{5}{|c|}{ MLE } \\
\hline & $\lambda$ & $\theta$ & $\beta$ & $\delta$ & $\alpha$ \\
\hline \multirow{2}{*}{10} & -0.5224 & 1.0175 & 3.7501 & 3.1175 & 2.6544 \\
\hline & 0.1270 & 0.1300 & 1.8205 & 0.1300 & 1.2817 \\
\hline \multirow{2}{*}{20} & -0.5058 & 1.0022 & 3.4466 & 3.0022 & 2.2391 \\
\hline & 0.0489 & 0.0507 & 1.4783 & 0.0507 & 0.3825 \\
\hline \multirow{2}{*}{30} & 0.5406 & -0.9949 & 2.9786 & 3.9949 & 2.1573 \\
\hline & 0.0299 & 0.0307 & 0.4971 & 0.0307 & 0.2298 \\
\hline \multirow{2}{*}{40} & -0.5634 & 0.9952 & 2.8352 & 3.9952 & 2.1215 \\
\hline & 0.0253 & 0.0224 & 0.3510 & 0.0224 & 0.1590 \\
\hline \multirow{2}{*}{50} & -0.6013 & 0.9954 & 2.7542 & 3.9954 & 2.0965 \\
\hline & 0.0181 & 0.0184 & 0.2685 & 0.0184 & 0.1252 \\
\hline \multirow{2}{*}{60} & -0.6101 & 0.9956 & 2.8154 & 3.9956 & 2.0804 \\
\hline & 0.0168 & 0.0148 & 0.2379 & 0.0148 & 0.0998 \\
\hline \multirow{2}{*}{70} & -0.6310 & 0.9966 & 2.7223 & 3.9966 & 2.0711 \\
\hline & 0.0119 & 0.0125 & 0.2175 & 3.0125 & 0.0872 \\
\hline \multirow{2}{*}{80} & -0.6627 & 0.9978 & 2.7700 & 3.9978 & 2.0553 \\
\hline & 0.0100 & 0.0106 & .0109 & 4.0106 & 0.0671 \\
\hline \multirow{2}{*}{90} & -0.6688 & 0.9992 & 2.6920 & 3.9992 & 2.0511 \\
\hline & 0.0089 & 0.0095 & 0.0089 & 3.0095 & 0.0619 \\
\hline \multirow{2}{*}{100} & -0.6502 & 0.9982 & 2.6518 & 3.9982 & 2.0471 \\
\hline & 0.0061 & 0.0087 & 0.0085 & 0.0087 & 0.0545 \\
\hline
\end{tabular}

From Table 2, it can be clearly observed that as sample size increases the mean square error decreases, which proves the consistency of the estimators.

\section{Concluding Remarks}

There has been a great interest among statisticians and applied researchers in constructing flexible lifetime models to facilitate better modelling of survival data. Consequently, a significant progress has been 
made towards the generalization of some well-known lifetime models and their successful application to problems in several areas. In this paper, we introduce a new generalization of the power Lindley distribution obtained by using the new generalization technique. We refer to the new model as the NGPL distribution and study some of its mathematical and statistical properties. We provide the pdf, the cdf, and the hazard rate function of the new model, explicit expressions for the moments. The model parameters are estimated by maximum likelihood and method of moment. The new model is compared with some models and provides consistently better fit than other lifetime models. We hope that the proposed distribution will serve as an alternative model to other models available in the literature.

\section{References}

[1] J. C. Ahuja and S. W. Nash, The generalized Gompertz-Verhulst family of distributions, Sankhya 29 (1967), 141-161.

[2] G. R. Aryal and C. P. Tsokos, Transmuted Weibull distribution: A generalization of the Weibull probability distribution, European Journal of Pure and Applied Mathematics 4(2) (2011), 89-102.

[3] A. Asgharzadeh, S. H. Bakouch, S. Nadarajah and L. Esmaeili, A new family of compound lifetime distributions, Kybernetika 50(1) (2014), 142-169.

[4] H. S. Bakouch, B. M. Al-Zahrani, A. A. Al-Shomrani, V. A. A. Marchi and F. Louzada, An extended Lindley distribution, J. Korean Stat. Soc. 41(1) (2012), 75-85.

[5] R. E. Barlow and F. Proschan, Statistical Theory or Reliability and Life Testing: Probability Models, Tobe with, (1981).

[6] H. W. Block and T. H. Savits, Burn-in, Statistical Science 12(1) (1997), 1-19.

[7] D. S. Chang, Optimal burn-in decision for products with a unimodal failure rate function, European Journal of Operational Research 126(3) (2000), 534-540.

[8] R. C. H. Cheng and N. A. K. Amin, Estimating parameters in continuous univariate distributions with a shifted origin, Journal of the Royal Statistical Society, Series B (Methodological) (1983), 394-403.

[9] I. Elbatal, Exponentiated modified Weibull distribution, Economic Quality Control 26(2) (2011), 189-200.

[10] M. E. Ghitany, D. K. Al-Mutairi, N. Balakrishnan and L. J. Al-Enezi, Power Lindley distribution and associated inference, Comput. Stat. Data Anal. 64 (2013), 20-33.

[11] M. E. Ghitany, B. Atieh and S. Nadarajah, Lindley distribution and its application, Math. Comput. Simulat. 78 (2008), 493-506. 
[12] M. E. Ghitany, F. Al-Qallaf, D. K. Al-Mutairi and H. A. Hussain, A two parameter weighted Lindley distribution and its applications to survival data, Math. Comput. Simulat. 81(6) (2011), 1190-1201.

[13] M. Greenwich, A unimodal hazard rate function and its failure distribution, Statistical Papers 33(1) (1992), 187-202.

[14] P. L. Gupta and R. C. Gupta, On the moments of residual life in reliability and some characterization results, Communications in Statistics-Theory and Methods 12 (1983) 449-461.

[15] R. D. Gupta and D. Kundu, Generalized exponential distributions, Australian \& New Zealand Journal of Statistics 41(2) (1999), 173-188.

[16] R. Jiang, P. Ji and X. Xiao, Ageing property of unimodal failure rate models, Reliability Engineering \& System Safety 79(1) (2003), 113-116.

[17] M. S. Khan and R. King, Transmuted modified Weibull distribution: A generalization of the modified Weibull probability distribution, European Journal of Pure and Applied Mathematics 6(1) (2013), 66-88.

[18] D. V. Lindley, Fiducial distributions and Bayes theorem, Journal of the Royal Statistical Society 20(1) (1958), 102-107.

[19] M. M. Mansour, M. A. Enayat, S. M. Hamed and M. S. Mohamed, A new transmuted additive Weibull distribution based on a new method for adding a parameter to a family of distributions, Accepted for JAMS.

[20] F. Merovci, Transmuted Lindley distribution, Int. J. Open Problems Compt. Math. 6(2) (2013), 63-72.

[21] F. Merovci, Transmuted generalized Rayleigh distribution, Journal of Statistics Applications and Probability 3(1) (2014), 9-20.

[22] F. Merovci and I. Elbatal, Transmuted Lindely-Geometric Distribution and its Application, J. Stat. Appl. Pro. 3(1) (2014), 77-91.

[23] G. S. Mudholkar and D. K. Srivastava, Exponentiated Weibull family for analyzing bathtub failure-rate data, IEEE Transactions on Reliability 42(2) (1993), 299-302.

[24] G. S. Mudholkar, D. K. Srivastava and M. Freimer, The exponentiated Weibull family: A reanalysis of the bus-motor-failure data, Technometrics 37(4) (1995), 436-445.

[25] S. Nadarajah, H. S. Bakouch and R. A. Tahmasbi, Generalized Lindley distribution, Sankhya B 73 (2011), 331-359.

[26] B. Oluyede and T. Yang, A new class of generalized Lindley distributions with applications, J. Stat. Comput. Simulat. (2014).

http://dx.doi.org/10.1080/00949655.2014.917308

[27] A. M. Sarhan and M. Zaindin, Modified Weibull distribution, Applied Sciences 11(1) (2009), 123-136. 
[28] W. T. Shaw and I. R. Buckley, The alchemy of probability distributions: Beyond Gram-Charlier expansions and a skew-kurtotic-normal distribution from a rank transmutation map, (2009).

arXivpreprint arXiv:0901.0434

[29] R. C. Team, R: A Language and Environment for Statistical Computing. R Foundation for Statistical Computing, Vienna, Austria, ISBN 3-900051-07-0, (2012).

[30] M. Xie and C. D. Lai, Stochastic Ageing and Dependence for Reliability, Springer, New York, 2006.

[31] T. Zhang, M. Xie, L. C. Tang and S. H. Ng, Reliability and modeling of systems integrated with firmware and hardware, International Journal of Reliability, Quality and Safety Engineering 12(03) (2005), 227-239. 